\title{
Abnormal intrinsic brain activities in stable patients with COPD: a resting-state functional MRI study
}

\author{
Wenjing Wang ${ }^{1, *}$ \\ Haijun $\mathrm{Li}^{2}$,* \\ Dechang Peng ${ }^{2}$ \\ Juan Luo' \\ Huizhen $\mathrm{Xin}^{2}$ \\ Honghui $Y^{2}$ \\ Jingjing $\mathrm{Yu}^{\prime}$
}

'Department of Respiratory, The First Affiliated Hospital of Nanchang University, Nanchang, China; ${ }^{2}$ Department of Radiology, The First Affiliated Hospital of Nanchang University, Nanchang, China

*These authors contributed equally to this work
This article was published in the following Dove Press journal: Neuropsychiatric Disease and Treatment

Objective: The majority of previous neuroimaging studies have reported both structural and functional changes in COPD, whereas the intrinsic low-frequency oscillations changes and the relationship between the abnormal brain regions and the clinical performances remain unknown. The present study was conducted with the aim of evaluating the intrinsic brain activity in COPD patients using the amplitude of low-frequency fluctuation (ALFF) method.

Methods: All participants, including 19 stable patients with COPD and 20 normal controls (NCs) matched in age, sex, and education, underwent resting-state functional MRI scans and performed cognitive function tests and respiratory functions tests. The local spontaneous brain activity was examined using the voxel-wise ALFF. Pearson's correlation analysis was used to investigate the relationships between the brain regions with altered ALFF signal values and the clinical features in COPD patients.

Results: Compared with the NCs, COPD patients showed significantly lower cognitive function scores. Also, lower ALFF areas in the cluster of the posterior cingulate cortex (PCC) and precuneus, as well as a higher ALFF area in the brainstem were also found in COPD patients. The mean ALFF values in the PCC, precuneus, and brainstem showed high sensitivity and specificity in operating characteristic curves analysis, which might have the ability to distinguish COPD from NCs. Meanwhile, the mean signal values of the lower ALFF cluster displayed significant positive correlations with $\mathrm{FEV}_{1} / \mathrm{FVC}$ proportion and significant negative correlation with $\mathrm{PaCO}_{2}$; the higher ALFF cluster showed significant positive correlation with $\mathrm{FEV}_{1}$ proportion in COPD.

Conclusion: According to the results of the present study, the COPD patients showed abnormal intrinsic brain activities in the precuneus, PCC, and brainstem, which might provide useful information to better understand the underlying pathophysiology of cognitive impairment.

Keywords: chronic obstructive pulmonary disease, amplitude of low-frequency fluctuation, functional magnetic resonance imaging, spontaneous brain activity, blood-oxygen-level dependent

\section{Introduction}

COPD is defined as "chronic airflow limitation, which is usually both progressive and associated with an abnormal inflammatory response of the lungs to noxious particles or gases". ${ }^{1}$ Moreover, it is a disease state characterized by airflow limitation that is not fully reversible. ${ }^{2}$ Although airflow obstruction is the most obvious manifestation of COPD, it is associated with many extra-pulmonary features that contribute to the morbidity, reduced quality of life, and increased mortality of this disease. ${ }^{3}$ Cognitive impairment is one of the most important extra-pulmonary manifestations in COPD. ${ }^{4-12}$
Correspondence: Jingjing Yu Department of Respiratory, The First Affiliated Hospital of Nanchang University, No 17, Yongwai Zheng Street, Donghu District, Nanchang 330006, Jiangxi Province, China

Tel +86 79l 88694457

Email yujingjing19740I2I@|63.com
Neuropsychiatric Disease and Treatment 2018:14 2763-2772

Dovepress f 10

http://dx.doi.org/10.2147/NDTSt80325 (c) (1) (8) $\odot 2018$ Wang et al. This work is published and licensed by Dove Medical Press Limited. The full terms of this license are avalable at hitps://www.dovepress.com/terms.php cc) hereby accept the Terms. Non-commercial uses of the work are permitted without any further permission from Dove Medical Press Limited, provided the work is properly attributed. For permission for commercial use of this work, please see paragraphs 4.2 and 5 of our Terms (https://www.dovepress.com/terms.php). 
Previous studies suggest that up to $77 \%$ of patients with both COPD and hypoxemia have some form of cognitive impairment. ${ }^{13}$ Cognitive impairment may be associated with the degree of lung function impairment in COPD. Moreover, cognitive impairment may lead to increased dyspnea and fatigue and result in incorrect use of inhaler devices, so might increase the exacerbation risk and could result in worse health outcome. ${ }^{4,5,14}$ According to a recent systemic review, COPD is currently the fourth and, by 2030, predicted to be the third leading cause of death worldwide. ${ }^{15}$ The main pathophysiologic mechanism of cognitive deficits from COPD, including oxidative stress, acute-phase response proteins, or in the activation levels of circulating cells and cytokines, ${ }^{2}$ for example, on the basis of previous study, in stable COPD patients, the expression of Mac-1 (CD11b/CD18) in circulating neutrophils was increased but with lower levels of soluble intercellular adhesion molecule (sICAM)-1; ${ }^{16}$ this remains poorly understood.

The majority of previous studies have demonstrated the structural and functional alterations related to COPD using advanced neuroimaging techniques. Examples of these changes include diminutions in gray matter density and gray matter volume, such as hippocampal atrophy. ${ }^{17,18}$ All restingstate networks, with the exception of the visual network, showed significantly greater activation in COPD. ${ }^{19}$ A SPECT study showed significantly lower brain perfusion in frontal and parietal lobes in COPD patients, and a significant change in brain metabolism in severe COPD patients has also been investigated. ${ }^{20-22}$ Resting-state functional MRI (rs-fMRI) is an effective way to investigate brain functions. Previously, the rs-fMRI was widely used in the areas of neurological and psychiatric diseases. However, recently, it's use has been increasing for the investigation of ongoing neuronal processes in COPD. Although the majority of previous neuroimaging studies have focused on the brain structural and functional changes in COPD patients, the changes in blood-oxygen-level dependent (BOLD) signals of regional spontaneous activity of COPD patients during resting state and their relationships with behavioral performances have not been fully understood.

Amplitude of low-frequency fluctuation (ALFF), an fMRI method, has been proven to have test-retest reliability, and has already been applied to patient studies investigating attention deficit hyperactivity disorder, ${ }^{23}$ early Alzheimer's disease, ${ }^{24}$ mild cognitive impairment, ${ }^{25}$ schizophrenia, ${ }^{26}$ and obstructive sleep apnea (OSA). ${ }^{27}$ It calculates the square root of the power spectrum in a low-frequency range $(0.01-0.08 \mathrm{~Hz})$ to detect the regional intensity of spontaneous fluctuations in BOLD signals. ${ }^{23,28,29}$
In the present study, we hypothesized that COPD patients would exhibit abnormal intrinsic local neural activities, which, in turn, would be related to clinical status and may provide additional information about brain dysfunction. So, we applied ALFF method to examine the low-frequency oscillations in COPD patients and its potential mechanisms and then correlate these results with behavioral performance in patients.

\section{Materials and methods Subjects}

The study population was composed of 19 stable COPD patients and 20 age-matched and years-of-education-matched normal controls (NCs). All of them were recruited at the Respiratory Department of The First Affiliated Hospital of Nanchang University from December 2017 to May 2018. All subjects were assessed by a detailed clinical interview and physical examination. In addition, they underwent pulmonary function testing to ensure all patients were in stable state (with no exacerbations during the past 8 weeks) or in stable state after therapy. The diagnoses and classification of COPD are based on the Global Strategy for the Diagnoses, Management, and Prevention of Chronic Obstructive Lung Disease 2017 Report, GOLD Executive Summary. ${ }^{30}$ Exclusion criteria were as follows: 1) OSA syndrome or insomnia; 2) mental disorders, such as epilepsy and brain tumor; 3) heart failure; 4) history of alcoholism and/or drugs; 5) dementia; 6) comorbidities, such as diabetes, liver failure and cardiovascular disease, and hypertension; 7) cerebral trauma or stroke or after brain operation; 8) unable to complete the evaluations of Montreal Cognitive Assessment (MoCA) and Mini-Mental State Examination (MMSE); and 9) contraindications to MRI, such as claustrophobia, metallic implants, or devices in the body.

The protocol was approved by The Human Research Ethics Committee of The First Affiliated Hospital of Nanchang University and performed according to the Declaration of Helsinki. All participants signed the informed consent.

\section{Pulmonary function}

The $\mathrm{FEV}_{1}, \mathrm{FEV}_{1} / \mathrm{FVC}$, and $\mathrm{FVC}$ were recorded. The subjects with $\mathrm{FEV}_{1} / \mathrm{FVC}<0.7$ and $\mathrm{FEV}_{1} \%$ pred $\geq 80 \%$ predicted were classified as mild COPD, $50 \% \leq \mathrm{FEV}_{1} \%$ pred $<80 \%$ were classified as moderate COPD, 30\% $\leq \mathrm{FEV}_{1} \%$ pred $<50 \%$ were classified as severe $\mathrm{COPD}$ and $\mathrm{FEV}_{1} \%$ pre $<30 \%$ were classified as extremely severe COPD. These indexes were obtained from a dry spirometer device (Erich Jaeger GmbH, Hoechberg, Germany) at 15 minutes after 
patients inhaling salbutamol $400 \mu \mathrm{g}$ (Ventolin; GlaxoSmithKline, London, UK).

\section{Arterial blood gas analysis}

Stat Profle Critical Care Xpress (Nova Biomedical, Waltham, MA, USA) was used to analyze the arterial blood in 30 minutes. Main indicators are as follows: Power of hydrogen, arterial partial pressure of oxygen $\left(\mathrm{PaO}_{2}\right)$, arterial partial pressure of carbon dioxide $\left(\mathrm{PaCO}_{2}\right)$, and blood oxygen saturation $\left(\mathrm{SaO}_{2}\right)$.

\section{Cognitive assessments}

The $\mathrm{MoCA}^{31}$ and the $\mathrm{MMSE}^{32}$ were used to assess cognitive functions. MoCA, a rapid screening instrument for mild cognitive impairment, can assess many different cognitive field, including naming, executive function, calculation, attention, language, memory, abstraction, and orientation. The total MoCA score was 30. A total MoCA score $<26$ indicates cognitive impairment, whereas a score $\geq 26$ means normal. If the length of schooling was $<12$ years, one point was added to the total score, so as to adjust for educational bias. MMSE can fully, accurately, and quickly reflect the intelligence state of the subjects and the degree of cognitive impairment. The highest score is 30. A total MMSE score between 27 and 30 is normal. The score $<27$ reveals cognitive impairment and the score between 21 and 26 means mild impairment, however, $10 \leq$ a score $\leq 20$ means moderate and a score $<9$ indicates severe. These two scales were conducted by two independent neuropsychologists. All participants completed the cognitive assessments as required. ${ }^{33}$

\section{Image acquisition}

In all subjects, MRI was performed at the same imaging session on 3.0 Tesla MRI system with 8-channel head coil (Siemens, Erlangen, Germany). First, conventional T1-weighted imaging (repetition time $[\mathrm{TR}]=250 \mathrm{~ms}$, echo time $[\mathrm{TE}]=2.46$, slices $=19$, slice thickness $=5 \mathrm{~mm}$, gap $=1.5 \mathrm{~mm}$, field of view $[\mathrm{FOV}]=220 \times 220 \mathrm{~mm}$ ) and T2-weighted imaging $(\mathrm{TR}=4,000 \mathrm{~ms}, \mathrm{TE}=113 \mathrm{~ms}$, slices $=19$, slice thickness $=5 \mathrm{~mm}$, gap $=1.5 \mathrm{~mm}, \mathrm{FOV}=220 \times 220 \mathrm{~mm}$ ) were collected. High-resolution T1-weighted images were acquired with a three-dimensional spoiled gradient-recalled echo sequence in a sagittal orientation with the parameters following: 176 images $(\mathrm{TR}=1,900 \mathrm{~ms}$; $\mathrm{TE}=2.26 \mathrm{~ms}$; thickness $=1.0 \mathrm{~mm}$; gap $=0 \mathrm{~mm}$; acquisition matrix $=256 \times 256$; FOV $=250 \times 250 \mathrm{~mm}^{2}$, flip angle $\left.=9^{\circ}\right) ; 240$ functional images $(\mathrm{TR}=2,000 \mathrm{~ms}$; $\mathrm{TE}=30 \mathrm{~ms}$; thickness $=4.0 \mathrm{~mm}$; gap $=1.2 \mathrm{~mm}$; acquisition matrix $=64 \times 64$; flip angle $=90^{\circ}$;
FOV $=230 \times 230 \mathrm{~mm}^{2} ; 30$ axial slices with gradient-recalled echo-planar imaging pulse sequence) were obtained.

\section{fMRI data preprocessing}

The preprocessing of rs-fMRI data was performed using Data Processing \& Analysis for Brain Imaging (DPABI) (http:// rfmri.org/DPABI) and Statistical Parametric Mapping (SPM8) (http://www.fil.ion.ucl.ac.uk/spm), which was run on the MATLAB2012a (Mathworks, Natick, MA, USA) platform. Included were the following: 1) the first ten volumes from each subject were discarded due to the signal reaching equilibrium and the participants' adaptation to the scanning noise.

2) Three-dimensional head motion correction was conducted for the remaining time points. None of the participants were removed according to the head motion criteria, which included a maximum $\operatorname{spin}\left(\mathrm{x}, \mathrm{y}\right.$, and $\mathrm{z}$ ) of $<2.0^{\circ}$ and a maximum cardinal direction displacement ( $\mathrm{x}, \mathrm{y}$, and $\mathrm{z}$ ) of $<2.0 \mathrm{~mm}$ and according to the standard of head motion, meaning that frame-wise displacement (FD) was $>2.5$ SDs based on the method of Van Dijk et al. ${ }^{34}$ 3) Anatomical and functional images were manually reoriented to the anterior commissure to achieve better registration, and structural images were co-registered to the functional images for each individual using a linear transformation. 4) The transformed structural images were segmented into gray matter, white matter, and cerebrospinal fluid using the new segmentation in SPM8. 5) The Diffeomorphic Anatomical Registration Through Exponentiated Lie Algebra tool was used to compute transformations from native space to Montreal Neurological Institute space, and re-sampled to $3 \times 3 \times 3 \mathrm{~mm}$ voxels. 6) The white matter signal, cerebrospinal fluid signal, and Friston 24-parameter were regressed from the time series of all voxels via linear regression. 7) Specific practice is that the remaining data were smoothed with a Gaussian kernel of $6 \times 6 \times 6 \mathrm{~mm}$ full width at half maximum. 8) A temporal filter $(0.01-0.08 \mathrm{~Hz})$ was performed to reduce the effect of lowfrequency drift and high-frequency noise.

\section{ALFF compulating}

The ALFF analysis was performed using the DPABI, ${ }^{35}$ which has been described in a previous study. ${ }^{27}$ Briefly, the time series for each voxel were transformed to the frequency domain, and the power spectrum was then obtained, the square root was calculated at each frequency of the power spectrum, and the averaged square root was obtained across $0.01-0.08 \mathrm{~Hz}$ at each voxel. This averaged square root was taken as the ALFF. For standardization, the ALFF of each voxel was z-transformed with Fisher's r-to-z transformation for subsequent group comparisons. The brain regions that 
demonstrated significant level of difference between groups were identified. These regions were classified as region of interests and saved as masks using the REST version 1.8 software (http://www.resting-fmri.Sourceforge.net). And then the mean ALFF value was extracted by averaging the ALFF values over all voxels for each COPD patient.

\section{Statistical analysis}

Demographic data like age, years of education, heart rate, systolic and diastolic blood pressure, arterial blood gas indexes, pulmonary function indicators, MoCA score, and MMSE score were tested using independent sample $t$-tests using the Statistical Package for the Social Sciences version 19.0 (IBM Corporation, Armonk, NY, USA).

The two-sample $t$-test was used to compare the z-ALFF values in each voxel of the two groups (two-tailed, voxel-level $P<0.01$; Gaussian random field theory correction, cluster-level $P<0.05$ ), using age, years of education, mean FD, and intracranial volume as nuisance covariates within the default gray matter mask. In addition, the receiver operating characteristic (ROC) was performed to verify the ALFF values, which altered in several brain regions and could be used as a biomarker to distinguish the COPDs from normal subjects. Finally, the Pearson's correlation analysis was used to investigate the relationship between the mean ALFF values in different brain regions and clinical performance in COPD patients. $P$-value $<0.05$ was considered to represent a significant difference.

\section{Results}

\section{Demographic and clinical data results}

Demographic and clinical characteristics of two groups are summarized in Table 1. The COPD patients had significantly lower scores for $\mathrm{SaO}_{2}, \mathrm{PaO}_{2}, \mathrm{FVC}, \mathrm{FEV}_{1}, \mathrm{FEV}_{1} / \mathrm{FVC}, \mathrm{MoCA}$, and MMSE, and had significantly higher scores for $\mathrm{PaCO}_{2}$ and pack-year (smoking history) than to NCs.

\section{ALFF alterations between COPD patients and NCs}

The mean ALFF values were showed in Table 2 and Figures 1 and 2. Which revealed that COPDs had a significant higher ALFF area in the brainstem, and lower ALFF areas in the posterior cingulate cortex (PCC) and precuneus compared to NCs. Meanwhile, the average ALFF z-values of the altered brain regions were showed in Figure 3.

\section{ROC curve results}

The alterations of the mean ALFF values in the PCC, precuneus, and brainstem in COPD might have the ability to distinguish COPD from NCs. In our present study, the area
Table I Demographic and clinical characteristics of COPD and NCs

\begin{tabular}{|c|c|c|c|}
\hline Characteristics & COPD $(n=19)$ & NCs $(n=20)$ & $P$-value \\
\hline Age (years) & $62.7 \pm 5.9$ & $60.8 \pm 6.3$ & 0.361 \\
\hline Male/female (n) & $14 / 5$ & $15 / 5$ & - \\
\hline Education (years) & $5.5 \pm 3.2$ & $6.3 \pm 4.5$ & 0.135 \\
\hline Disease duration (years) & $4.5 \pm 5.6$ & - & - \\
\hline Pack-years & $32.2 \pm 21.7$ & $8.5 \pm 8.9$ & $<0.001$ \\
\hline $\mathrm{SaO}_{2}(\%)$ & $95.5 \pm 2.6$ & $97.8 \pm 1.8$ & 0.008 \\
\hline $\mathrm{PaO}_{2}(\mathrm{~mm} \mathrm{Hg})$ & $82.6 \pm 16.5$ & $98 \pm 19.5$ & $<0.001$ \\
\hline $\mathrm{PaCO}_{2}(\mathrm{~mm} \mathrm{Hg})$ & $49.3 \pm 8.0$ & $38.5 \pm 4.3$ & $<0.001$ \\
\hline $\begin{array}{l}\text { Respiratory rate } \\
\text { (times/min) }\end{array}$ & $19.5 \pm 0.6$ & $18.5 \pm 1.2$ & 0.654 \\
\hline FVC (\% predicted) & $67.5 \pm 19.9$ & $96.5 \pm 15.8$ & $<0.00 \mathrm{I}$ \\
\hline FEV $(\%$ predicted $)$ & $46.1 \pm 20.6$ & $97.1 \pm 16.9$ & $<0.001$ \\
\hline $\mathrm{FEV} / \mathrm{FVC}(\%)$ & $55.8 \pm 16.3$ & $81.2 \pm 8.3$ & $<0.001$ \\
\hline $\mathrm{MoCA}$ & $18.4 \pm 4.3$ & $26.4 \pm 3.2$ & $<0.001$ \\
\hline MMSE & $22.4 \pm 3.6$ & $27.2 \pm 2.2$ & $<0.001$ \\
\hline Intracranial volume $\left(\mathrm{cm}^{3}\right)$ & $\mathrm{I}, 540.20 \pm \mathrm{I} 10.49$ & 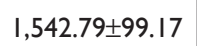 & 0.895 \\
\hline
\end{tabular}

Abbreviations: $\mathrm{FEV}_{1}$, forced expiratory volume in one second; $\mathrm{FVC}$, forced vital capacity; MMSE, Mini-mental State Examination; MoCA, Montreal Cognitive Assessment; $\mathrm{NCs}$, normal controls; $\mathrm{PaCO}_{2}$, arterial partial pressure of carbon dioxide; $\mathrm{PaO}_{2}$, partial pressure of oxygen; $\mathrm{SaO}_{2}$, blood oxygen saturation.

under the curve of the PCC and precuneus were 0.9 and 0.932 (Figure 4A), respectively; the area under the curve of the brainstem was 0.821 (Figure 4B). Furthermore, the sensitivity and specificity of the PCC were $70.0 \%$ and $94.7 \%$, respectively. Likewise, the sensitivity and specificity of the precuneus were $75.0 \%$ and $94.7 \%$, and of the brainstem were $68.4 \%$ and $90.0 \%$, respectively, which showed high-efficiency discriminant function of the altered average ALFF values.

\section{Correlation results}

In the present study, the mean ALFF values in PCC displayed significant positive correlation with $\mathrm{FEV}_{1} / \mathrm{FVC}(r=0.523$, $P=0.021$ ), and significant negative correlation with $\mathrm{PaCO}_{2}$ ( $r=-0.509, P=0.026$ ), the average values of ALFF in precuneus suggested a strong negative correlation with $\mathrm{PaCO}_{2}$ $(r=-0.628, P=0.004)$ in COPD patients. However, the higher ALFF in brainstem displayed positive correlation with $\mathrm{FEV}_{1} \%$ predicted $(r=0.518, P=0.023$ ) (Figure 5). However, no correlations were observed between abnormal ALFF value and lower scores on the neuropsychological tests.

\section{Discussion}

In the present study, ALFF analysis was used to investigate the intrinsic low-frequency oscillations amplitude of stable COPD patients and revealed abnormal neural activities in several brain regions. COPD patients had a significant higher ALFF in the brainstem, and showed lower ALFF areas in the PCC and precuneus significantly compared with NCs. Moreover, we also found that the higher ALFF area had 
Table 2 Two-sample $t$-test differences between COPD and NCs using ALFF method $(P<0.0$ I, GRF-corrected for multiple comparisons at a cluster level of $P<0.05$ )

\begin{tabular}{l|l|l|l|l|l|l|l|l}
\hline Conditions & Brain areas & R/L & BA & \multicolumn{2}{l|}{ MNI } & \multicolumn{2}{l}{ Cluster (voxel) } \\
\cline { 5 - 7 } & & & & $\mathbf{X}$ & $\mathbf{Y}$ & $\mathbf{Z}$ & & \\
\hline COPD $<$ NCs & Posterior cingulate cortex & R/L & 23 & 3 & -54 & 27 & 84 & -4.593 \\
COPD $<$ NCs & Precuneus & R/L & 19 & 3 & -54 & 45 & 84 & -4.469 \\
COPD $>$ NCs & Brainstem & R/L & $/$ & -3 & -36 & -27 & 79 & 4.458 \\
\hline
\end{tabular}

Abbreviations: ALFF, amplitude of low-frequency fluctuation; GRF, Gaussian random field; MNI, Montreal Neurological Institute; NCs, normal controls; R/L, right/left hemicerebrum.

a positive correlation with $\mathrm{FEV}_{1} \%$ predicted, whereas the lower ALFF areas revealed negative correlation with $\mathrm{PaCO}_{2}$ and positive correlation with $\mathrm{FEV}_{1} / \mathrm{FVC}$. Furthermore, ROC analysis suggested that the mean ALFF values in the PCC, precuneus, and brainstem could serve as markers to separate individuals with stable COPD patients from NCs.

All areas of the brain have a high level of organized default functional activity. The intrinsic functional activity in assessing brain behavior relationships is considered to be important; this activity implied the existence of a default mode. ${ }^{36}$ At present, the default mode network (DMN) is the most studied in brain networks and the most active. ${ }^{37}$ In our results, the PCC and precuneus are the core areas of the DMN, which are associated with the extraction of episodic memory collection, evaluation of information, mind wandering or daydreaming, and attention processing. ${ }^{38}$ Esser et $\mathrm{al}^{39}$ found that the gray matter decreased in the PCC and were relevant for the processing of dyspnea in COPD patients. Similarly, a recent study found that the changes in ratings of breathlessness word cues positively correlated with changes in activity in the cingulate cortes. ${ }^{40}$ Meanwhile, Zhang et $\mathrm{al}^{41}$ considered that chronic hypoxia has also been

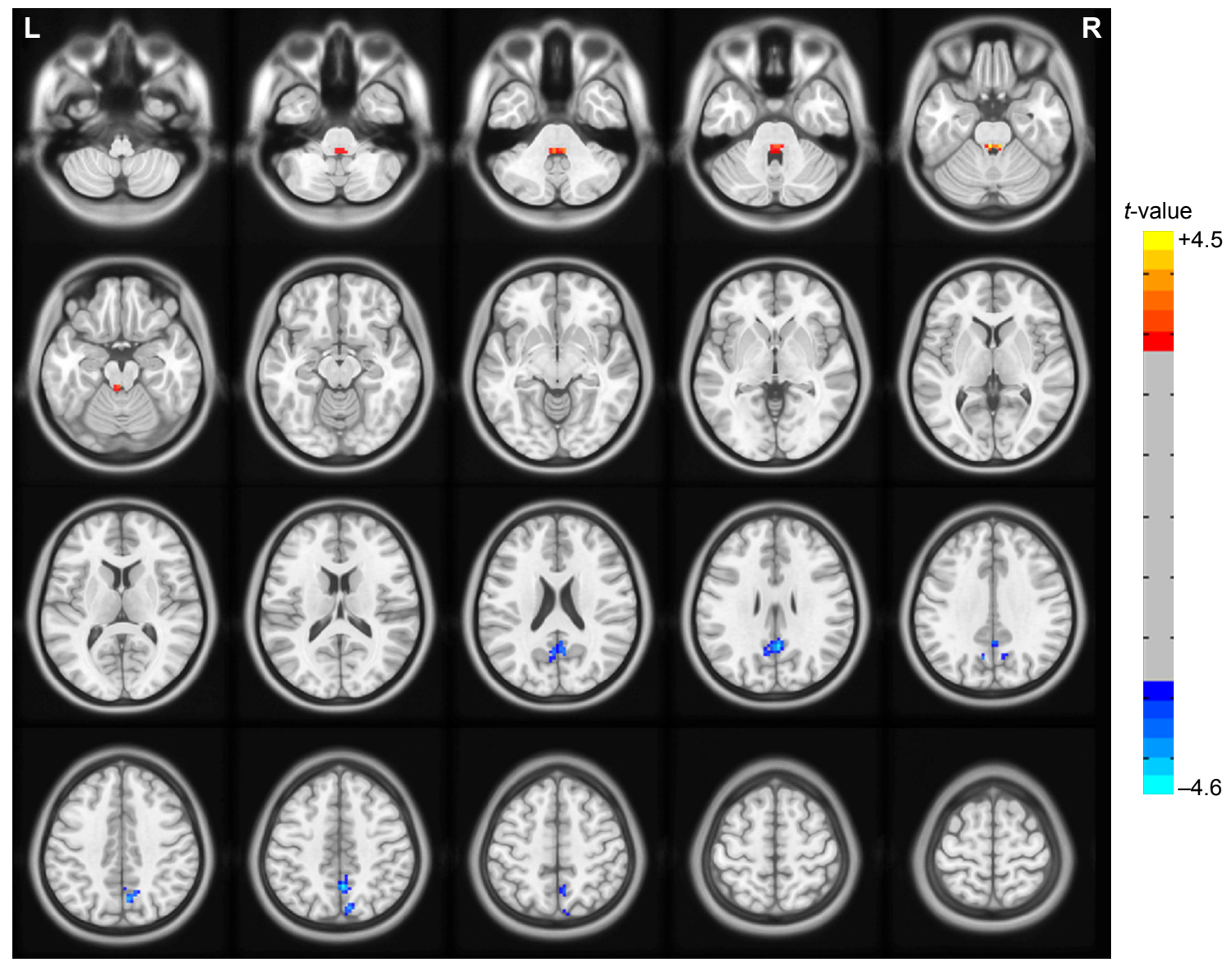

Figure I Differences in ALFF between COPD patients and NCs groups (two-tailed, voxel-level $P<0.01$; GRF correction, cluster-level $P<0.05$ ) are shown in axial position. Note: The red or yellow denotes higher ALFF values, and the blue areas indicate lower ALFF values, respectively.

Abbreviations: ALFF, amplitude of low-frequency fluctuation; GRF, Gaussian random field; NCs, normal controls; R/L, right/left hemicerebrum. 

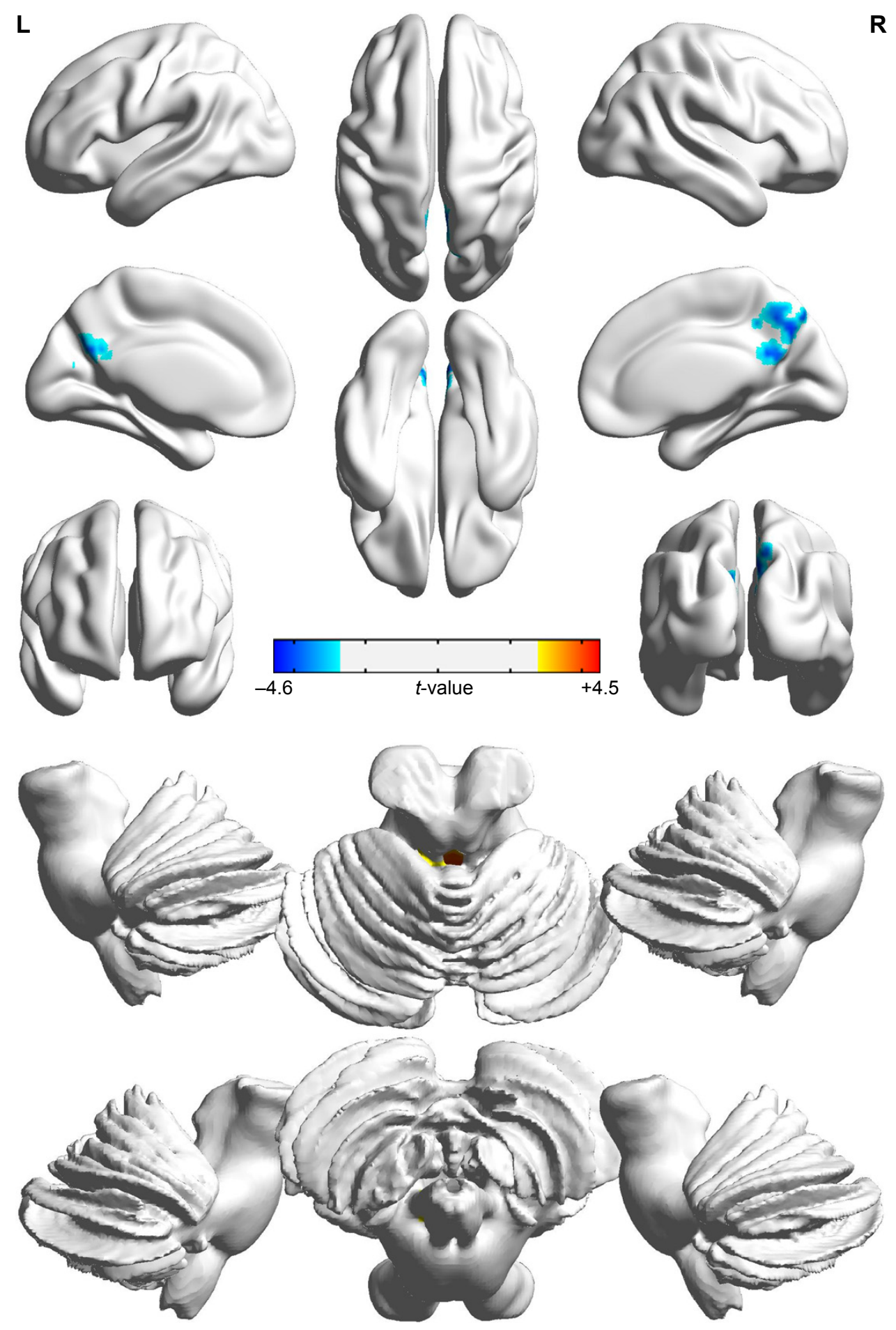

Figure 2 Significant activity differences were observed in PCC, precuneus, and brainstem between COPD patients and NCs groups (two-tailed, voxel-level $P<0.01$; GRF correction, cluster-level $P<0.05$ ) are shown in three-dimensional image.

Abbreviations: GRF, Gaussian random field; NCs, normal controls; PCC, posterior cingulate cortex; R/L, right/left hemicerebrum.

proven to reduce neural excitability in PCC. The impairment of functional connectivity in PCC was also investigated in asymptomatic carotid stenosis patients. ${ }^{42}$ Corfield et al ${ }^{43}$ thought that hypercapnia was associated with widespread BOLD signal increases. Marshall et a ${ }^{44}$ deemed that mild carbon dioxide can diminish brain functional connectivity in many brain areas, because hypercapnia aggravate intraischemic acidosis and subsequent brain damage. Consistent with previous researches, our study found that COPD patients showed lower ALFF value in the PCC, which negatively cor- 


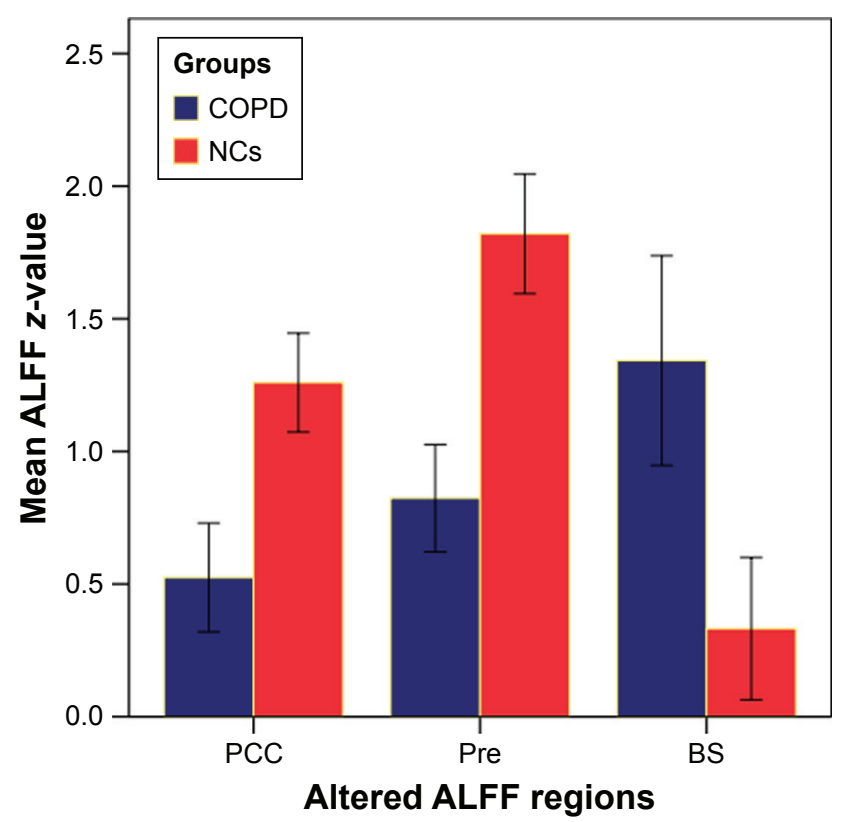

Figure 3 Mean ALFF signal values for altered regional brain areas between COPD patients and NCs.

Abbreviations: ALFF, amplitude of low-frequency fluctuation; BS, brainstem; NCs, normal controls; PCC, posterior cingulate cortex; Pre, precuneus.

related with $\mathrm{PaCO}_{2}$ and positively correlated with the $\mathrm{FEV}_{1} /$ FVC, suggesting that hypoxia may be an important factor for the PCC dysfunction in COPD.

The precuneus is a part of the $\mathrm{DMN},{ }^{46}$ and plays a key role in the processing of visual-spatial information, ${ }^{47}$ episodic memory retrieval, ${ }^{48}$ working memory, ${ }^{49}$ and consciousness. ${ }^{50}$ A previous study has revealed that OSA patients showed reduced perfusion in the right precuneus in association with hypoxemia and hypercapnia. ${ }^{51}$ COPD is similar to OSA, our study found lower-ALFF in the precuneus displayed negative correlated with $\mathrm{PaCO}_{2}$ in $\mathrm{COPD}$, we speculate that hypercapnia may be cause precuneus damage.

The brainstem automatic network maintains and controls the breath..$^{52}$ Yu et al ${ }^{53}$ found that patients with COPD showed high excitability of the brainstem respiratory neuronal, but the motor cortex area exhibited no connectivity with the brainstem, either at rest or during inspiratory loading, so high neuronal excitability responsible for decreased functional connectivity of the motor cortex is probably due to the chronic respiratory overload, impaired sensory afferents from the respiratory muscles and lungs, and hypoxia. Goossens et $\mathrm{al}^{54}$ pointed out that the patients of panic attacks show increased brainstem activation in response to hypercapnia, which is likely due to increased neural sensitivity to $\mathrm{CO}_{2}$ at brainstem level. Similarly, the present study showed the higher ALFF in brainstem is positively correlated with the $\mathrm{FEV}_{1} \%$ predicted, which may be a kind of adaptive compensatory response.

\section{Limitations}

There are several limitations that should be paid attention to. First of all, the present study was a case-control study, and

\section{A}

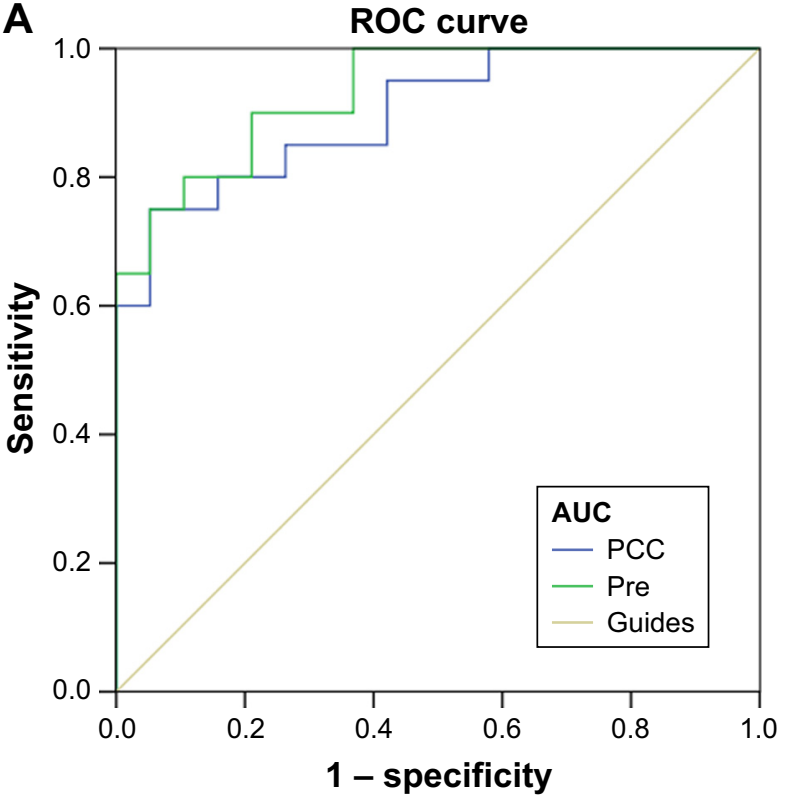

B

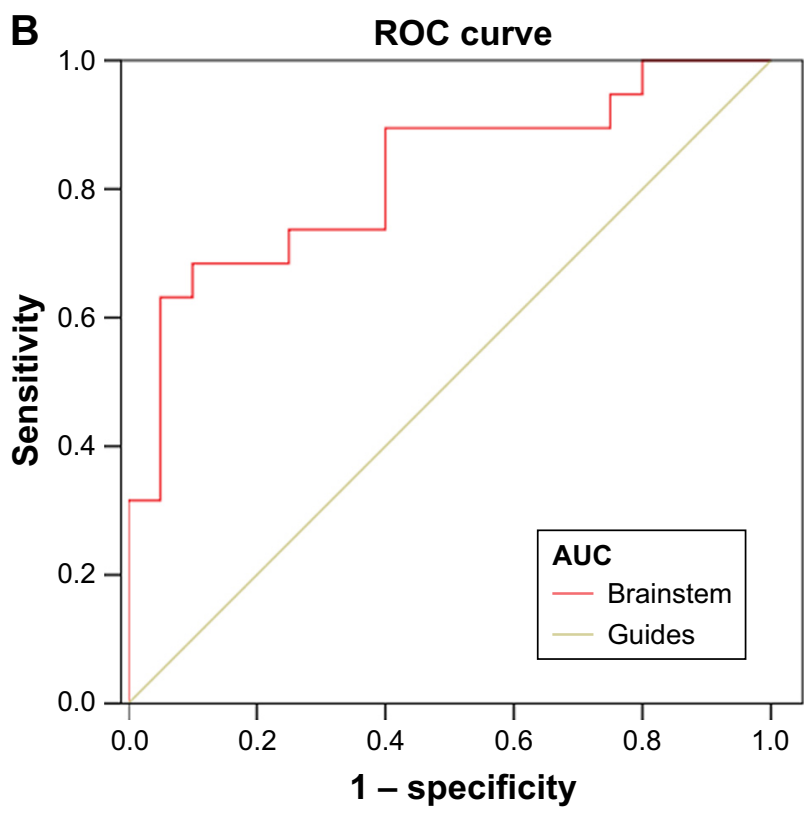

Figure 4 ROC curve analysis of the mean ALFF values for altered brain regions.

Notes: The AUCs were 0.9 for the PCC and 0.932 for the precuneus (A). The AUCs were 0.821 for the brainstem (B). The sensitivity and specificity of the PCC were $70.0 \%$ and $94.7 \%$, respectively. The sensitivity and specificity of the precuneus were $75.0 \%$ and $94.7 \%$, respectively. The sensitivity and specificity of the brainstem were $68.4 \%$ and $90.0 \%$, respectively.

Abbreviations: ALFF, amplitude of low-frequency fluctuation; AUC, area under the curve; PCC, posterior cingulate cortex; ROC, receiver operating characteristic. 

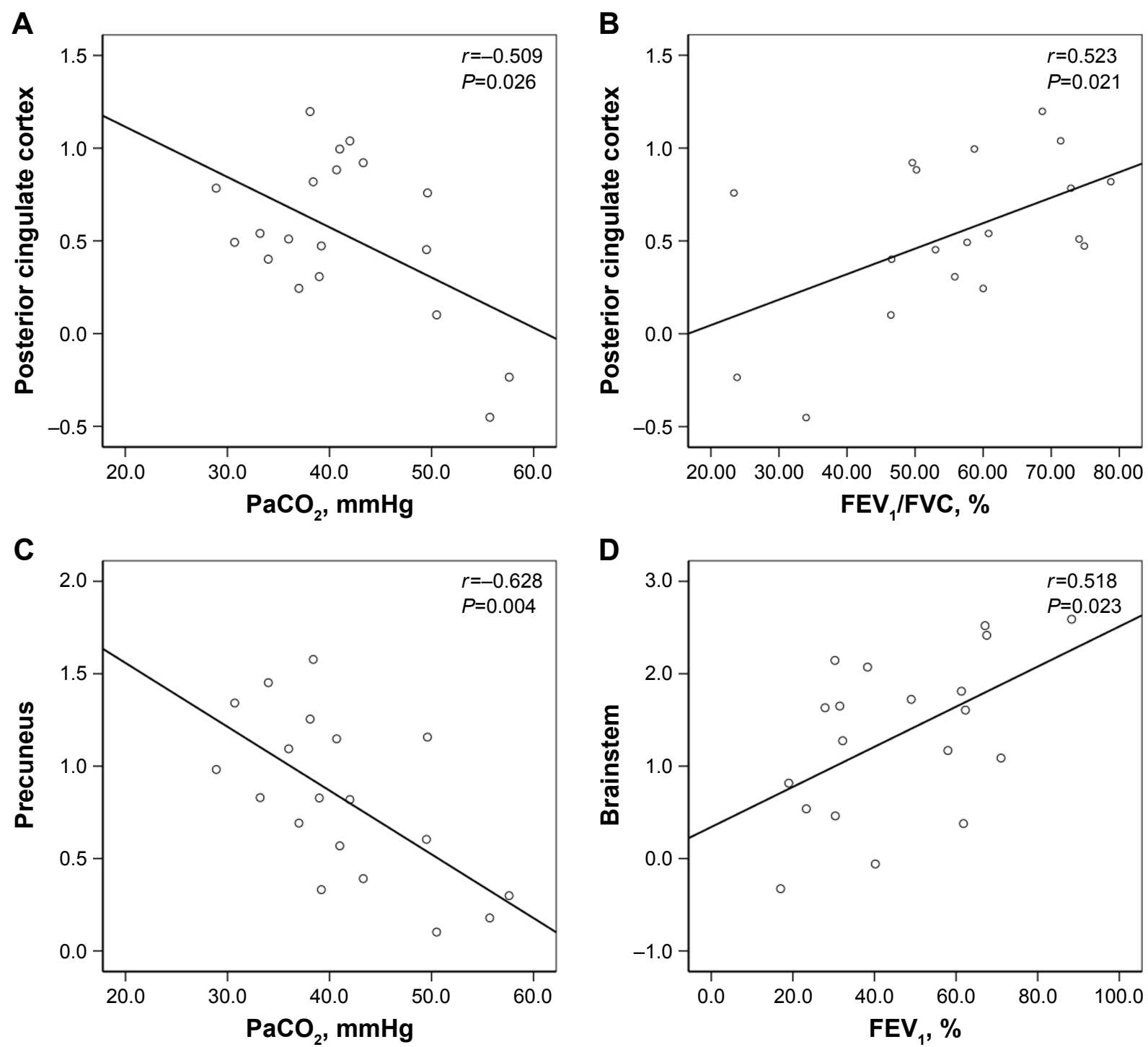

Figure 5 Significant correlation was observed between the ALFF values in the PCC (A, B), precuneus (C), brainstem (D) and the clinical parameters $\left(\mathrm{PaCO}_{2}\right.$, FEV $/$ FVC, and $\mathrm{FEV}_{1}$ ) in the stable COPD patient group.

Abbreviations: ALFF, amplitude of low-frequency fluctuation; $\mathrm{PaCO}_{2}$, partial pressure of carbon dioxide; PCC, posterior cingulate cortex.

we cannot completely rule out the effect of medication used for COPD therapy, although it is unlikely that the medication affected our results. Second, the study comprised a relatively small population, so the severity of the disease could not be considered. In addition, some inflammatory factors, such as C-reactive protein, interleukin (IL)-6, and IL-8 in patients with COPD play a key role in the neural activity. ${ }^{55-59}$ Given these limitations, we prepare for further study the effects of disease classification, treatment and serum inflammatory factors on intrinsic activities.

\section{Conclusion}

Our current study has showed that the intrinsic brain activity alterations exist in COPD patients. Particularly, the ALFF signal values in PCC, precuneus, and brainstem are strongly relevant to the clinical performance. In addition, the mean ALFF values could serve as biomarkers to distinguish COPD patients from NCs, which may provide evidence of an intrinsic plasticity link with hypoxia in COPD patients.

\section{Acknowledgment}

This study was supported by the Natural Science Foundation of China (grant no. 81560285, 81860307), the Natural Science Foundation Project of Jiangxi, China (grant no. 20171BAB205070, 20181ACB20023), Education Department Project of Jiangxi provincial, China (grant no. 700544006), Department of Health Project and Jiangxi provincial, China (grant no. 20181039) and Graduate Innovation Foundation of Jiangxi, China (grant no. YC2015-S082). 


\section{Disclosure}

The authors report no conflicts of interest in this work.

\section{References}

1. Singh B, Parsaik AK, Mielke MM, et al. Chronic obstructive pulmonary disease and association with mild cognitive impairment: the Mayo Clinic Study of Aging. Mayo Clin Proc. 2013;88(11):1222-1230.

2. Wouters E, Creutzberg EC, Schols A. Systemic effects in COPD. Chest. 2002:127S-130S.

3. Ortapamuk H, Naldoken S. Brain perfusion abnormalities in chronic obstructive pulmonary disease: comparison with cognitive impairment. Ann Nucl Med. 2006;20(2):99-106.

4. Meek PM, Lareau SC, Anderson D. Memory for symptoms in COPD patients: how accurate are their reports? Eur Respir J. 2001 18(3):474-481.

5. Cleutjens F, Spruit MA, Ponds R, et al. Cognitive functioning in obstructive lung disease: results from the United Kingdom biobank. J Am Med Dir Assoc. 2014;15(3):214-219.

6. Derkacz M, Mosiewicz J, Myśliński W. Cognitive dysfunction in patients with chronic obstructive pulmonary disease. Wiad Lek. 2007; 60(3-4):143-147.

7. Fujimoto S, Kobayashi S, Yoshikawa T, Hirata K. Cognitive function in patients with chronic obstructive pulmonary disease. Nihon Rinsho. 2014;72(4):721-725.

8. Cleutjens FA, Janssen DJ, Gijsen C, Dijkstra JB, Ponds RW, Wouters EF. Cognitive impairment in patients with COPD: a review. Tijdschr Gerontol Geriatr. 2014;45(1):1-9.

9. López Torres I, Torres-Sánchez I, Martín Salvador A, Ortiz Rubio A, Rodríguez Alzueta E, Valenza MC. Cognitive impairment, nutritional status and clinical profile in chronic obstructive pulmonary disease. Nutr Hosp. 2014;30(5):1152-1159.

10. Sarınç Ulaşlı S, Oruç S, Günay E, et al. Effects of COPD on cognitive functions: a case control study. Tuberk Toraks. 2013;61(3):193-199.

11. Incalzi RA, Gemma A, Marra C, Muzzolon R, Capparella O, Carbonin P Chronic obstructive pulmonary disease. An original model of cognitive decline. Am Rev Respir Dis. 1993;148(2):418-424.

12. Incalzi RA, Chiappini F, Fuso L, Torrice MP, Gemma A, Pistelli R Predicting cognitive decline in patients with hypoxaemic COPD. Respir Med. 1998;92(3):527-533.

13. Grant I, Heaton RK, Mcsweeny AJ, Adams KM, Timms RM. Neuropsychologic findings in hypoxemic chronic obstructive pulmonary disease. Arch Intern Med. 1982;142(8): 1470.

14. Dodd JW, Charlton RA, van den Broek MD, Jones PW. Cognitive dysfunction in patients hospitalized with acute exacerbation of COPD. Chest. 2013;144(1):119-127.

15. Baird C, Lovell J, Johnson M, Shiell K, Ibrahim JE. The impact of cognitive impairment on self-management in chronic obstructive pulmonary disease: A systematic review. Respir Med. 2017;129:130-139.

16. Riise GC, Larsson S, Löfdahl CG, Andersson BA, Gc R. Circulating cell adhesion molecules in bronchial lavage and serum in COPD patients with chronic bronchitis. Eur Respir J. 1994;7(9):1673-1677.

17. Li J, Fei GH. The unique alterations of hippocampus and cognitive impairment in chronic obstructive pulmonary disease. Respir Res. 2013; 14(1):140

18. Cleutjens F, Ponds R, Spruit MA, et al. The Relationship between Cerebral Small Vessel Disease, Hippocampal Volume and Cognitive Functioning in Patients with COPD: An MRI Study. Front Aging Neurosci. 2017;9:88

19. Dodd JW, Chung AW, van den Broek MD, Barrick TR, Charlton RA, Jones PW. Brain structure and function in chronic obstructive pulmonary disease: a multimodal cranial magnetic resonance imaging study. $\mathrm{Am}$ $J$ Resp Crit Care. 2012;186(3):240.

20. Shim TS, Lee JH, Kim SY, et al. Cerebral metabolic abnormalities in COPD patients detected by localized proton magnetic resonance spectroscopy. Chest. 2001;120(5):1506-1513.
21. Ortapamuk H, Naldoken S. Brain perfusion abnormalities in chronic obstructive pulmonary disease: comparison with cognitive impairment. Ann Nucl Med. 2006;20(2):99-106.

22. Hu X, Wang $\mathrm{H}, \mathrm{Tu} \mathrm{Y}$, et al. Alterations of the default mode network and cognitive impairments in patients with chronic obstructive pulmonary disease. Int J Chron Obstruct Pulmon Dis. 2018;13:519-528.

23. Zang YF, He Y, Zhu CZ, et al. Altered baseline brain activity in children with ADHD revealed by resting-state functional MRI. Brain Dev. 2007;29(2):83-91.

24. He Y, Wang L, Zang Y, et al. Regional coherence changes in the early stages of Alzheimer's disease: a combined structural and resting-state functional MRI study. Neuroimage. 2007;35(2):488-500.

25. Han Y, Wang J, Zhao Z, et al. Frequency-dependent changes in the amplitude of low-frequency fluctuations in amnestic mild cognitive impairment: a resting-state fMRI study. Neuroimage. 2011;55(1): 287-295.

26. Hoptman MJ, Zuo XN, Butler PD, et al. Amplitude of low-frequency oscillations in schizophrenia: a resting state fMRI study. Schizophr Res. 2010;117(1):13-20.

27. Li HJ, Dai XJ, Gong HH, Nie X, Zhang W, Peng DC. Aberrant spontaneous low-frequency brain activity in male patients with severe obstructive sleep apnea revealed by resting-state functional MRI. Neuropsychiatr Dis Treat. 2015;11:207-214.

28. Kiviniemi V, Jauhiainen J, Tervonen O, et al. Slow vasomotor fluctuation in fMRI of anesthetized child brain. Magn Reson Med. 2000; 44(3):373-378.

29. Lu H, Zuo Y, Gu H, et al. Synchronized delta oscillations correlate with the resting-state functional MRI signal. Proc Natl Acad Sci U S A. 2007;104(46):18265-18269.

30. Vogelmeier CF, Criner GJ, Martinez FJ, et al. Global Strategy for the Diagnosis, Management, and Prevention of Chronic Obstructive Lung Disease 2017 Report. GOLD Executive Summary. Am J Respir Crit Care Med. 2017;195(5):557-582.

31. Nasreddine ZS, Phillips NA, Bédirian V, et al. The Montreal Cognitive Assessment, MoCA: a brief screening tool for mild cognitive impairment. J Am Geriatr Soc. 2010;53(4):695-699.

32. Folstein MF, Folstein SE, Mchugh PR. "State" "mini-Mental". "Minimental state". A practical method for grading the cognitive state of patients for the clinician. J Psychiatr Res. 1975;12(3):189-198.

33. Orth M, Kotterba S, Duchna K, et al. [Cognitive deficits in patients with chronic obstructive pulmonary disease (COPD)]. Pneumologie. 2006; 60(10):593-599. Romanian.

34. van Dijk KR, Sabuncu MR, Buckner RL. The influence of head motion on intrinsic functional connectivity MRI. Neuroimage. 2012;59(1): 431-438.

35. Yan CG, Wang XD, Zuo XN, Zang YF. DPABI: Data Processing \& Analysis for (Resting-State) Brain Imaging. Neuroinformatics. 2016; 14(3):339-351.

36. Raichle ME, Snyder AZ. A default mode of brain function: a brief history of an evolving idea. Neuroimage. 2007;37(4):1083-1090.

37. Raichle ME, Macleod AM, Snyder AZ, Powers WJ, Gusnard DA, Shulman GL. A default mode of brain function. Proc Natl Acad Sci U S A. 2001;98(2):676-682.

38. Mason MF, Norton MI, van Horn JD, Wegner DM, Grafton ST, Macrae CN. Wandering minds: the default network and stimulusindependent thought. Science. 2007;315(5810):393-395.

39. Esser RW, Stoeckel MC, Kirsten A, et al. Structural Brain Changes in Patients With COPD. Chest. 2016;149(2):426-434.

40. Herigstad M, Faull OK, Hayen A, et al. Treating breathlessness via the brain: changes in brain activity over a course of pulmonary rehabilitation. Eur Respir J. 2017;50(3):1701029.

41. Zhang J, Chen J, Yu Q, et al. Alteration of spontaneous brain activity in COPD patients. Int J Chron Obstruct Pulmon Dis. 2016;11(1):1713-1719.

42. Cheng HL, Lin CJ, Soong BW, et al. Impairments in cognitive function and brain connectivity in severe asymptomatic carotid stenosis. Stroke. 2012;43(10):2567-2573. 
43. Corfield DR, Murphy K, Josephs O, Adams L, Turner R. Does hypercapnia-induced cerebral vasodilation modulate the hemodynamic response to neural activation? Neuroimage. 2001;13(6 Pt 1):1207-1211.

44. Marshall O, Uh J, Lurie D, Lu H, Milham MP, Ge Y. The influence of mild carbon dioxide on brain functional homotopy using resting-state fMRI. Hum Brain Mapp. 2015;36(10):3912-3921.

45. Kurihara J, Katsura K, Siesjö BK, Wieloch T. Hyperglycemia and hypercapnia differently affect post-ischemic changes in protein kinases and protein phosphorylation in the rat cingulate cortex. Brain Res. 2004;995(2):218-225

46. Utevsky AV, Smith DV, Huettel SA. Precuneus is a functional core of the default-mode network. J Neurosci. 2014;34(3):932-940.

47. Cavanna AE, Trimble MR. The precuneus: a review of its functional anatomy and behavioural correlates. Brain. 2006;129(Pt 3):564-583.

48. Riggins T, Geng F, Blankenship SL, Redcay E. Hippocampal functional connectivity and episodic memory in early childhood. Dev Cogn Neurosci. 2016;19(C):58-69.

49. van Snellenberg JX, Slifstein M, Read C, et al. Dynamic shifts in brain network activation during supracapacity working memory task performance. Hum Brain Mapp. 2015;36(4):1245-1264.

50. Cavanna AE, Trimble MR. The precuneus: a review of its functional anatomy and behavioural correlates. Brain. 2006;129(Pt 3):564-583.

51. Baril AA, Gagnon K, Arbour C, et al. Regional cerebral blood flow during wakeful rest in older subjects with mild to severe obstructive sleep apnea. Sleep. 2015;38(9):1439-1449.
52. Hess A, Yu L, Klein I, et al. Neural mechanisms underlying breathing complexity. PLoS One. 2013;8(10):e75740

53. Yu L, de Mazancourt M, Hess A, et al. Functional connectivity and information flow of the respiratory neural network in chronic obstructive pulmonary disease. Hum Brain Mapp. 2016;37(8):2736-2754.

54. Goossens L, Leibold N, Peeters R, et al. Brainstem response to hypercapnia: a symptom provocation study into the pathophysiology of panic disorder. J Psychopharmacol. 2014;28(5):449-456.

55. Mukandala G, Tynan R, Lanigan S, O'Connor JJ. The Effects of Hypoxia and Inflammation on Synaptic Signaling in the CNS. Brain Sci. 2016;6(1):6.

56. Gan WQ, Man SF, Senthilselvan A, Sin DD. Association between chronic obstructive pulmonary disease and systemic inflammation: a systematic review and a meta-analysis. Thorax. 2004;59(7):574-580.

57. O'Connor MF, Irwin MR, Wellisch DK. When grief heats up: proinflammatory cytokines predict regional brain activation. Neuroimage. 2009;47(3):891-896.

58. Inagaki TK, Muscatell KA, Irwin MR, Cole SW, Eisenberger NI. Inflammation selectively enhances amygdala activity to socially threatening images. Neuroimage. 2012;59(4):3222-3226.

59. Muscatell KA, Moieni M, Inagaki TK, et al. Exposure to an inflammatory challenge enhances neural sensitivity to negative and positive social feedback. Brain Behav Immun. 2016;57:21-29.
Neuropsychiatric Disease and Treatment

\section{Publish your work in this journal}

Neuropsychiatric Disease and Treatment is an international, peerreviewed journal of clinical therapeutics and pharmacology focusing on concise rapid reporting of clinical or pre-clinical studies on a range of neuropsychiatric and neurological disorders. This journal is indexed on PubMed Central, the 'PsycINFO' database and CAS,

\section{Dovepress}

and is the official journal of The International Neuropsychiatric Association (INA). The manuscript management system is completely online and includes a very quick and fair peer-review system, which is all easy to use. Visit http://www.dovepress.com/testimonials.php to read real quotes from published authors. 